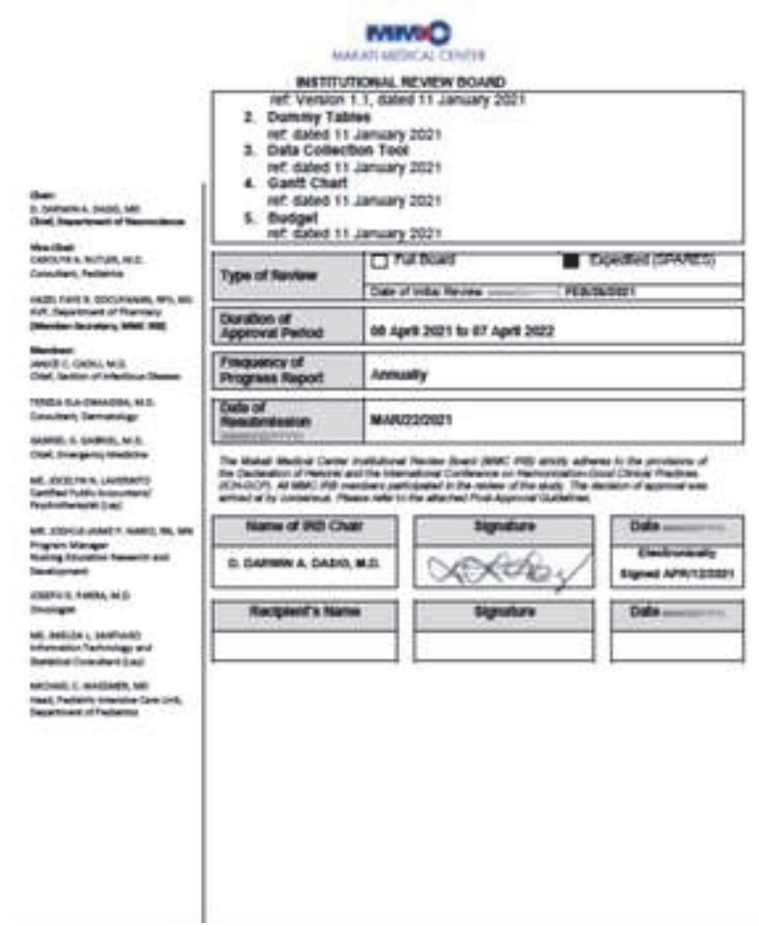

Abstract 182 Figure 2

Methods This was a retrospective, analytical, observational, cohort type of study conducted through chart review of kidney patients at a tertiary care center approved by the IRB (Protocol Number 2021-003).

Results A total of 355 patients were included in this study where the median age was 64 years old. More than half of the cohort (57.18\%) was male. Most of the subjects (93\%) were classified as ASA category III. The most common surgical procedure for securing vascular access was arteriovenous fistula creation (39.72\%) while the most common BPB performed was axillary approach (59.15\%), followed by supraclavicular approach (19.44\%), infraclavicular approach (14.93\%) and interscalene approach (6.48\%).

All four techniques recorded no incidence of inadequate block and only low incidence for need of rescue pain medication for infraclavicular $(0.47,95 \%$ CI: $0-10.01)$ and axillary (0.47, 95\% CI: 0-2.62) blocks. This resulted to a low overall incidence of need of rescue pain medication at $0.56,95 \% \mathrm{CI}$ : $0-2.02$.

Conclusions Axillary, infraclavicular and supraclavicular BPB are appropriate in procedures involving the wrist, distal arm and elbow (i.e., AV fistula creation). In procedures involving the arm such as AV graft creation and thrombectomy, infraclavicular, supraclavicular and interscalene BPB may be utilized.

\section{A CASE REPORT OF ERECTOR SPINAE PLANE BLOCK WITH CONTINUOUS INFUSION FOR MULTIPLE RIB FRACTURES}

J Cleland*, M Hulgur. Royal Albert Edward Infirmary, Wigan, UK

10.1136/rapm-2021-ESRA. 183
Background and Aims Rib fractures cause significant pain and respiratory complications ${ }^{1}$. Common analgesic techniques include opiates and thoracic epidurals, both have significant side effects ${ }^{2}$. Erector spinae plane (ESP) block with catheter insertion has been used as an alternative due to its ability to provide analgesia with a potentially better side effect profile ${ }^{3,4}$. We describe a case of a 74 year old gentleman with a significant smoking history and COPD who had suffered trauma and fractured ribs $8-11$ on his right side.

Methods To perform the ESP block we injected local anaesthetic and then inserted a catheter between the erector spinae muscle and transverse processes (figure 1). A bolus dose of 30 $\mathrm{ml} 0.25 \%$ levobupivacaine was injected followed by infusion of bupivacaine $0.125 \% 10 \mathrm{ml} / \mathrm{hr}$. This was later increased to $15 \mathrm{ml} / \mathrm{hr}$ due to some patient discomfort.

Results Prior to the ESP block the patient had required 44 PCA doses in $24 \mathrm{hrs}$, afterwards he required 10 doses in 24 hrs. Pain scores improved from a baseline level of $8 / 10$ with episodes of $10 / 10$ prior to block, to a baseline level of $0 / 10$ with episodes of $2 / 10$ afterwards. Prior to ESP block he was unable to use the incentive spirometer due to pain, the following day he was able to use the incentive spirometer without any pain (figure 2).

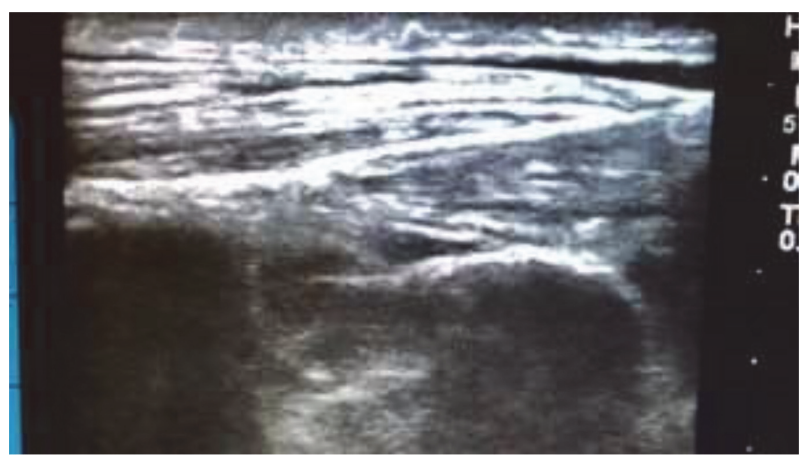

Abstract 183 Figure 1

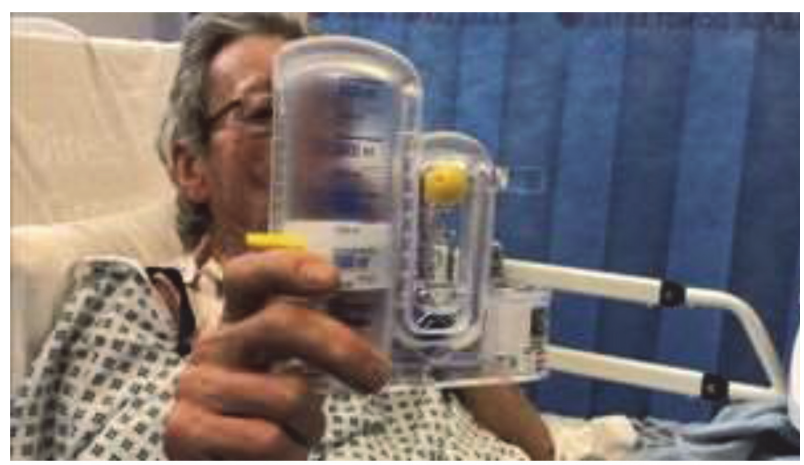

Abstract 183 Figure 2

Conclusions The ESP block both reduced the patients pain symptoms and improved his respiratory function. The patient much preferred the ESP block to the PCA he had been using before. ESP block is a useful tool in the management of pain from rib fractures. 